\title{
Hollow core photonic bandgap fibre for truly single mode operation
}

\author{
F. Poletti, M. N. Petrovich, A van Brakel and D. J. Richardson \\ Optoelectronics Research Centre, University of Southampton, Southampton, SO17 1BJ, United Kingdom, \\ Email:frap@orc.soton.ac.uk
}

\begin{abstract}
Based on extensive numerical simulations we fabricate a 3-cell core photonic bandgap fibre. Robust single mode guidance is observed even after metre length propagation.
\end{abstract}

\section{Introduction}

Hollow core photonic bandgap fibres (PBGFs) allow light guidance in a low refractive index dielectric, enabling significant advances in applications such as high power laser delivery, pulse compression, gas sensing and gas-based nonlinear optics, amongst others. It is well known that the most widely employed PBGF, incorporating a core with 7 missing cells (7c) is multimoded [1]. An even greater number of guided modes are supported from the larger core 19 missing cell core (19c) variants reported. In both cases, a number of studies have demonstrated that by using a sufficient length of fibre, a nearly single mode output can be achieved; this is attributed to a differential loss between fundamental mode (FM) and higher order modes (HOMs) [2]. However, this approach fails for device applications requiring a single mode output in a short length of fibre. A recent numerical study suggested that by reducing the fibre core to only 3 omitted capillaries (3c) a fibre that is single moded at all wavelengths within the bandgap can, in principle, be realised [3].

In this study we extensively compare the modal properties of realistic PBGFs with a 3c, 7c and 19c core. Encouraged by the predictions of truly single mode behaviour for a $3 \mathrm{c}$ PBGF, we then fabricated what we believe to be the first such fibre and experimentally observed the anticipated robust single mode guidance.

\section{Simulation results and fabricated fibre}

To calculate the modal characterisation of a 3c, 7c and 19c PBGFs we employ a full vector finite element method. Following previous SEM analysis of fabricated 7c-fibres operating at $1500 \mathrm{~nm}$ we based the 3 fibres on an idealised but realistic cladding and core structure with the following characteristics: 7 rings of holes, hole-to-hole spacing $\Lambda=$ $3.8 \mu \mathrm{m}$, relative hole diameter $\mathrm{d} / \Lambda=0.97$, and radius of the circular sector rounding the hexagonal holes Dc/d $=0.4$. For each fibre we evaluated the main optical properties of all the air-guided modes. A selection of these results, showing the effective index $\left(\mathrm{n}_{\mathrm{eff}}\right)$ and the normalised surface scattering coefficient (F-factor, [4]) is shown in Fig.1; Table 1 compares the main properties of the FMs. Note that a careful choice of the core boundary thickness [5] allows surface mode-free FM operation at all wavelengths and in all three cases.

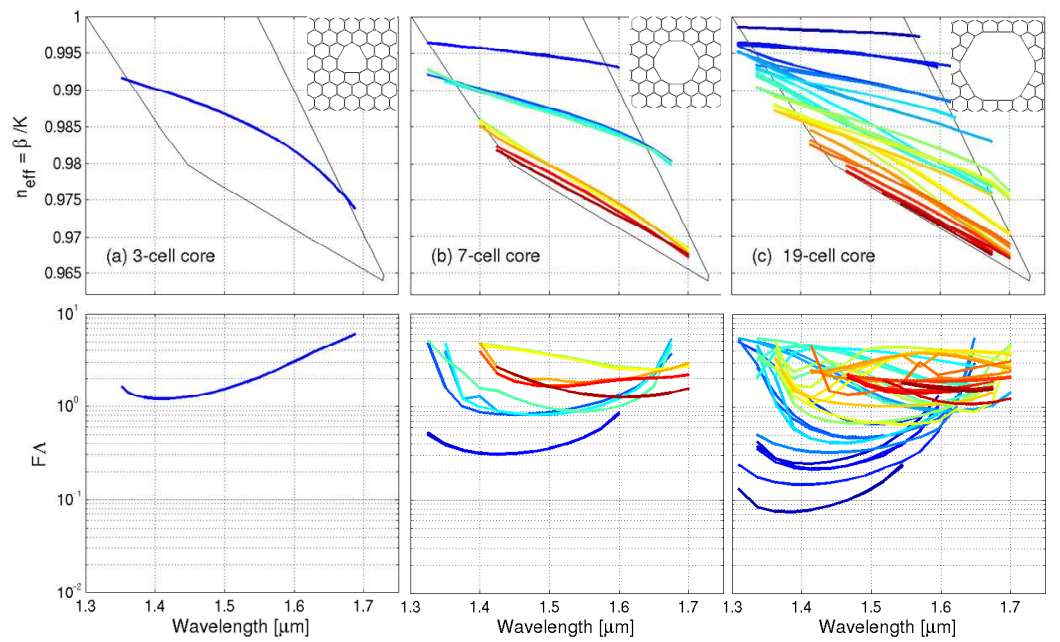

Fig.1. Simulated effective index (top) and F- $\Lambda$ (bottom) of all the modes guided in the hollow core for (a) $3 \mathrm{c}$, (b) $7 \mathrm{c}$ and (c) $19 \mathrm{c}$ fibres.

The simulations show that fibre $7 \mathrm{c}$ guides 12 modes $\left(\mathrm{LP}_{01}, \mathrm{LP}_{11}, \mathrm{LP}_{02}\right.$ and $\left.\mathrm{LP}_{21}\right)$, while up to 40 modes are guided in fibre 19c around $1570 \mathrm{~nm}$. Both F-factor and CL increase with the mode order. Modes guided close to the lower edge of the bandgap present CLs around $100 \mathrm{~dB} / \mathrm{m}$ or larger, and therefore are not likely to be observed in practice. 
However, the first $4 \mathrm{HOMs}\left(\mathrm{LP}_{11}\right)$ of the $7 \mathrm{c}$ fibre still present a minimum CL of less than $0.01 \mathrm{~dB} / \mathrm{m}$, while more than 25 modes of the 19c fibre have CL lower than $1 \mathrm{~dB} / \mathrm{m}$ and therefore should all be observed over metre-long lengths. Note also that the increase in F-factor (proportional to the surface scattering loss) with mode order is quite modest, only $\sim 2-3$ times between $\mathrm{LP}_{01}$ and $\mathrm{LP}_{11}$ modes and $\sim 4-7$ times between $\mathrm{LP}_{01}$ and $\mathrm{LP}_{21}$ modes. These results suggest that the modal differential loss cannot fully account for the nearly single mode output observed in tens-ofmetres long PBGFs. We believe that the low coupling efficiency of HOMs to a linearly polarised Gaussian source, optimised for the launch of the FM, also plays an important role here. As a result, the quality of the fibre output for fibres $7 \mathrm{c}$ and $19 \mathrm{c}$ critically depends on both fibre length and input coupling conditions.

\begin{tabular}{|l|ccc|ccc|}
\cline { 2 - 7 } \multicolumn{1}{c|}{} & \multicolumn{3}{c|}{ Properties across the bandgap } & \multicolumn{3}{c|}{ at 1500 nm } \\
\hline Power in glass (\%) & 3c & 7c & 19c & 3c & 7c & 19c \\
$\mathbf{A}_{\text {eff }}\left[\boldsymbol{\mu m}^{\mathbf{2}}\right]$ & $27-80$ & $0.5-1.2$ & $0.13-0.6$ & 2.9 & 0.7 & 0.2 \\
$\gamma\left[\mathbf{W}^{-1} \mathbf{k m}^{-\mathbf{1}}\right]$ & $0.013-0.7$ & $0.0027-0.004$ & 0.001 & 0.02 & 0.003 & 0.001 \\
\hline
\end{tabular}

Table 1 - Calculated optical properties of the FMs of the three PBGF

This problem can be avoided altogether with fibre 3c, where only two degenerate fundamental modes appear to be guided inside the bandgap. We estimated that the smaller core radius results in an increased percentage of power in the glass (up to $3 \%$ ) and therefore nonlinearity $(\gamma)$, while the CL increases by nearly two orders of magnitude as compared to $7 \mathrm{c}$, suggesting that an additional ring of holes may be beneficial. The F-factor calculation (Fig.1, bottom row) shows that the truly single mode guidance in fibre $3 \mathrm{c}$ is achieved at the cost of an estimated 4-5 times increase in (surface scattering) loss as compared to a 7c, while the 7c should be approximately 3 times as lossy as the $19 \mathrm{c}$. Based on these values and on the lowest loss so far reported for a $7 \mathrm{c}$ PBGF (13 dB/km [1]), we predict a minimum loss for the $3 \mathrm{c}$ of around $60 \mathrm{~dB} / \mathrm{km}$, which is tolerable for applications requiring only meter lengths of fibre. Based on these encouraging results we fabricated a $3 \mathrm{c}$ fibre (Fig. 2(a)).

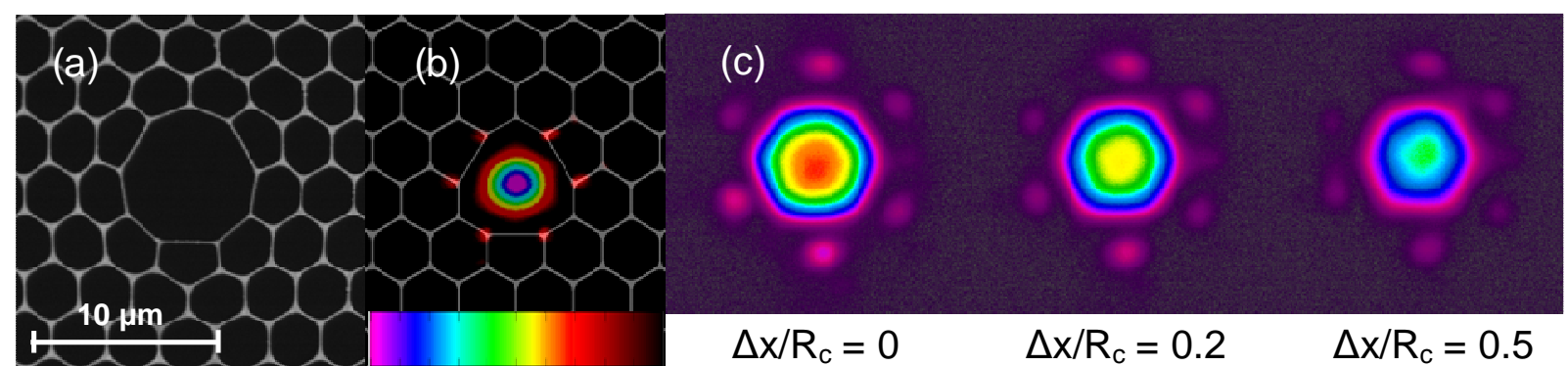

Fig.2. (a) SEM of the fabricated fibre; (b) simulated FM; (c) measured mode profiles after a $2 \mathrm{~m}$ long $3 \mathrm{c}$ PBGF, for different offsets $\Delta \mathrm{x}$ of the input launch conditions ( $\mathrm{R}_{\mathrm{c}}$ is the core radius).

The fabricated fibre exhibits a $\sim 250 \mathrm{~nm}$ wide transmission window centred at $1550 \mathrm{~nm}$, with only one surface mode visible at the short edge of the bandgap. The long wavelength edge is significantly less steep than that observed for fibres $7 \mathrm{c}$ and 19c, which correlates well with the dispersion curve in Fig 1 . The measured transmission loss of 0.2 $\mathrm{dB} / \mathrm{m}$, was limited by fabrication issues. Preliminary experimental measurements suggest that the fibre remains single mode even when a smaller-than-optimum, offset, spot size is used to launch light into short lengths of the fibre. Using an arc fusion splicer, we were able to consistently obtain good strength splices with a SMF with a total loss of $\sim 2 \mathrm{~dB}$ per splice (as compared to $\sim 1 \mathrm{~dB}$ for a $7 \mathrm{c}$ fibre, with a better matched $\mathrm{A}_{\mathrm{eff}}$ ).

\section{Conclusion}

Extensive numerical simulations of realistic PBGFs suggest that a truly single mode fibre can be obtained with a $3 \mathrm{c}$ core. We report the first fabrication of a $3 \mathrm{c}$ PBGF and preliminary near field measurements that support the argument of strong single mode guidance. We believe that the truly single mode guidance may prove particularly beneficial in gas cells or interferometer based applications.

\section{References}

[1] C. M. Smith et al., "Low-loss hollow-core silica-air photonic bandgap fiber", Nature 424, 657-659 (2003).

[2] G. Bowmans et al., "Properties of a hollow-core photonics bandgap fiber at $850 \mathrm{~nm}$ wavelength", Opt. Express 11, 1613-1620 (2003).

[3] T. Murao et al., "Realization of Single-Moded Broadband Air-Guiding Photonic Bandgap Fibers", IEEE PTL 18, 1666-1668 (2006).

[4] P. J. Roberts et al., "Ultimate low loss of hollow-core photonic crystal fibers", Opt. Express 13, 236-244 (2005).

[5] R. Amezcua-Correa et al, "Optimizing the usable bandwidth and loss through core design in realistic hollow-core photonic bandgap fibers", Opt. Express 14, 7974-7985 (2006). 